\title{
Halo Mass Estimation for Galaxy Groups: The Role Of Magnitude Gaps
}

\author{
Yi Lu ${ }^{1}$, Xiaohu Yang ${ }^{1,2}$ and Shiyin Shen ${ }^{1}$ \\ ${ }^{1}$ Key Laboratory for Research in Galaxies and Cosmology, \\ Shanghai Astronomical Observatory, Nandan Road 80, Shanghai 200030, China \\ email: luyi@shao.ac.cn \\ ${ }^{2}$ Center for Astronomy and Astrophysics, \\ Shanghai Jiao Tong University, Shanghai 200240, China \\ email: xyang@sjtu.edu.cn
}

\begin{abstract}
We find that for the galaxy groups, the luminosity gap between the brightest and the subsequent brightest member galaxies in a halo (group) can be used to significantly reduce the scatter in the halo mass estimation based on the luminosity of the brightest galaxy alone. These corrections can significantly reduce the scatter in the halo mass estimations by $\sim 50 \%$ to $\sim 70 \%$ in massive halos.
\end{abstract}

\section{Introduction}

Galaxy groups provide an important step in understanding processes in galaxy formation and cosmology. There are many methods to identify galaxy groups. Yang et al.(2005a) developed a halo-based group finder which has been successfully applied to 2dFGRS (Yang et al. 2005a), SDSS DR4 and DR7 (Yang et al. 2007). One of the key steps in the halo-based group finder is the estimation of halo masses of candidate galaxy groups. Usually, group total luminosity (e.g. Yang et al. 2005a; 2007) is considered to be a reliable halo mass indicator. Unfortunately, for shallow and high redshift surveys, only a few brightest member galaxies can be observed in each dark matter halo. In case the survey volume is difficult to calculate because of the bad survey geometry, the halo mass estimation based on the total luminosity may become unachievable.

To estimate the halo masses for poor galaxy systems, one may make use of the centralhost halo relation. As shown in Yang et al. (2008), for massive halos $L_{c} \propto M_{h}^{\sim 0.25}$, the typical scatter is about 0.15 . The central (or the brightest) galaxy alone cannot provide a reliable estimation. Thus we improve this relation by using luminosity gap, defined as $\log L_{\text {gap }}=\log L_{c}-\log L_{i}$, where $L_{i}$ is the luminosity of the i-th brightest member galaxies.

\section{Overview}

We make use of four sets of mock galaxy catalogs obtained from conditional luminosity function, the subhalo abundance, and semi-analytical model respectively. In Fig. 1, blue open circles show the median and the $68 \%$ confidence levels (error bars) of halo masses, $\log M_{h}$, as a function of central galaxy luminosity $\log L_{c}$. We fit the median $M_{h}\left(L_{c}\right)$ relations with the following functional form:

$$
\log M_{h}=\exp \left(\log L_{c}-\log M_{a}\right)+\log M_{b} .
$$

To tighten the errorbars by using luminosity gap, we formally write

$$
\log M_{h}\left(L_{c}, L_{\text {gap }}\right)=\log M_{h}\left(L_{c}\right)+\Delta \log M_{h}\left(L_{c}, L_{\text {gap }}\right),
$$




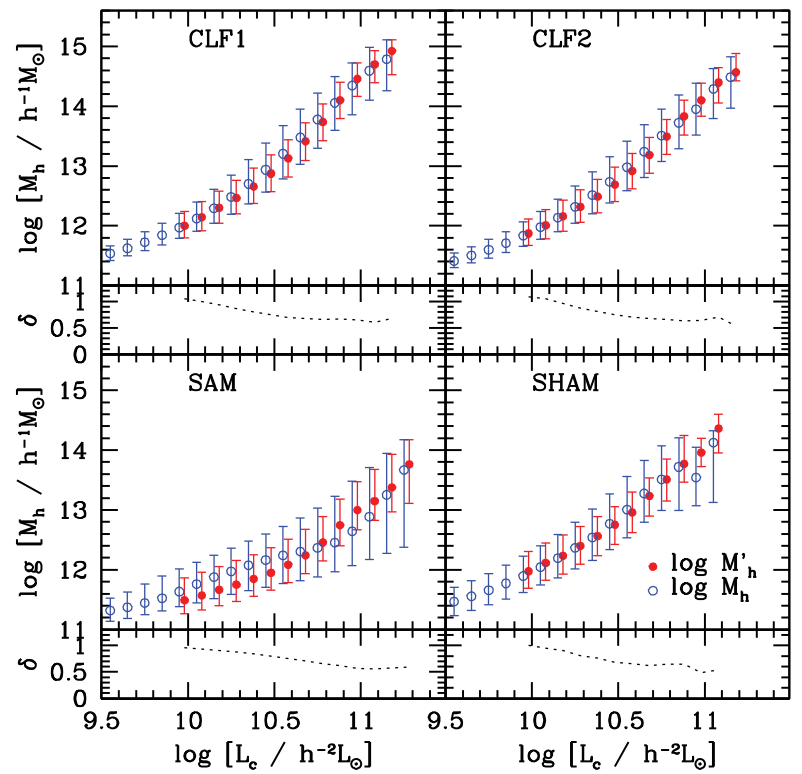

Figure 1. The comparison between the scatter in the original $\log M_{h}\left(L_{c}\right)$ relation and the new $\log M_{h}\left(L_{c}, L_{\text {gap }}\right)$ model.

where the first term on the right side is the empirical relation described by Eq. (2.1). And the 'luminosity gap' here, defined as the luminosity ratio between the central and the second brightest member galaxies in the same dark matter halo, $L_{\text {gap }}=L_{c} / L_{2}$. We use the following functional form to model $\Delta \log M_{h}\left(L_{c}, L_{\text {gap }}\right)$,

$$
\Delta \log M_{h}\left(L_{c}, L_{\text {gap }}\right)=\eta_{a} \exp \left(\eta_{b} \log L_{\text {gap }}\right)+\eta_{c},
$$

where parameters $\eta_{a}, \eta_{b}$ and $\eta_{c}$ may all depend on $L_{c}$ :

$$
\begin{aligned}
& \eta_{a}\left(L_{c}\right)=\exp \left(\log L_{c}-\beta_{1}\right) \\
& \eta_{b}\left(L_{c}\right)=\alpha_{2}\left(\log L_{c}+\beta_{2}\right), \\
& \eta_{c}\left(L_{c}\right)=-\left(\log L_{c}-\beta_{3}\right)^{\gamma_{3}}
\end{aligned}
$$

which in total has five free parameters. For comparison, we define a 'pre-corrected' halo mass

$$
\log M_{h}^{\prime}=\log M_{h}-\Delta \log M_{h}\left(L_{c}, L_{\text {gap }}\right),
$$

and check if the scatter in the $M_{h}^{\prime}\left(L_{c}\right)$ relation is significantly reduced relative to that in the $M_{h}\left(L_{c}\right)$. If the correction by $\Delta \log M_{h}\left(L_{c}, L_{\text {gap }}\right)$ were perfect, the scatter in the $M_{h}^{\prime}\left(L_{c}\right)$ would be reduced to 0 .

In Fig. 1, we define the ratio between the corrected and original errorbars as $\delta$ in the sub-panels. It is clear that the scatter in $M_{h}^{\prime}\left(L_{c}\right)$ is significantly reduced, especially for massive halos/groups where the scatter is reduced by about $50 \%$.

\section{References}

Yang, X., Mo, H. J., van den Bosch, F. C., \& Jing, Y. P. 2005a, MNRAS, 356, 1293

Yang, X., Mo, H. J., van den Bosch, F. C., et al. 2007, ApJ, 671, 153

Yang, X., Mo, H. J., \& van den Bosch, F. C. 2008, ApJ, 676, 248 